\title{
Dificuldades enfrentadas pelo portador de hipertensão arterial sistêmica à adesão ao tratamento na Estratégia Saúde da Família
}

\section{Difficulties faced by patients with systemic arterial hypertension and adherence to treatment in the Family Health Strategy}

\author{
Thays Jorgeana Alexandre e Silva \\ Bacharelada em Enfermagem, Faculdade Santa Maria, E-mail: thays.jorgeana@ hotmail.com \\ Elvira Uchôa dos Anjos de Almeida \\ Enfermeira, Docente da Faculdade da Faculdade Santa Maria, E-mail: elvira.uchoa@hotmail.com \\ Symara Abrantes Albuquerque de Oliveira Cabral \\ Doutoranda em Ciências da Saúde, Faculdade de Ciências Médicas da Santa Casa de São Paulo, E-mail: symara_abrantes@ hotmail.com \\ Carla Heloisa de Alencar Figueiredo \\ Bacharelada em Farmácia, FASP, E-mail: carla.heloisa@ hotmail.com
}

\begin{abstract}
Resumo: A hipertensão arterial sistêmica (HAS) é considerada um grave problema de saúde pública no Brasil e no Mundo. Sendo considerada um dos maiores fatores de risco para o surgimento de doenças cardiovasculares, renais e cerebrovasculares dentre outras. A terapia anti-hipertensiva é extremamente eficaz em reduzir a pressão arterial. Contudo, o percentual de pacientes que alcançam os alvos terapêuticos é baixo. A não adesão ao tratamento é um dos mais importantes problemas enfrentados, neste sentido, objetivou-se identificar as dificuldades enfrentadas pelo portador de hipertensão arterial sistêmica a adesão do tratamento na Estratégia Saúde da Família (ESF), esta responsável pela Atenção Primária à Saúde, seno ainda o centro das redes de atenção à saúde. Trata-se de um estudo exploratório, descritivo com abordagem quantitativa com a população de 4.772 hipertensos cadastrados no programa HiperDia na secretaria de saúde do município de Cajazeiras, a amostra foi composta de 100 hipertensos. Os resultados demostraram prevalência do sexo feminino (65\%), a média de idade ficou entre 59 anos (35\%). Quanto aos principais motivos que impedem a adesão ao tratamento $89 \%$ referiram falta de conhecimento. Antecedentes familiares o predomínio foi entre irmãos $66 \%$. Existe um déficit de adesão ao tratamento, especialmente por usuárias do sexo feminino, que relatam falta de conhecimento, revelando a necessidade de melhores estratégias de atuação no tocante à educação em saúde.
\end{abstract}

Palavras-chave: Atenção Primária à Saúde; Hipertensão; Terapêutica.

\begin{abstract}
Systemic arterial hypertension (SAH) is considered a serious public health problem in Brazil and in the World. It is considered one of the major risk factors for cardiovascular, renal and cerebrovascular diseases, among others. Antihypertensive therapy is extremely effective in lowering blood pressure. However, the percentage of patients reaching therapeutic targets is low. Non-adherence to treatment is one of the most important problems faced. In this sense, the objective was to identify the difficulties faced by patients with systemic arterial hypertension, adherence to treatment in the Family Health Strategy (ESF), which is responsible for Primary Health Care, The center of health care networks. This is an exploratory, descriptive study with a quantitative approach with the population of 4,772 hypertensive patients enrolled in the HiperDia program at the department of health of the municipality of Cajazeiras, the sample was composed of 100 hypertensive patients. The results showed female prevalence (65\%), mean age was 59 years $(35 \%)$. Regarding the main reasons that prevent adherence to treatment, $89 \%$ reported lack of knowledge. Family history The predominance was between siblings $66 \%$. The data show that there is a deficit of adherence to treatment, especially by female users, who report a lack of knowledge, revealing the need for better strategies in health education.
\end{abstract}

Keywords: Primary Health Care; Hypertension; Therapy. 


\section{INTRODUÇÃO}

As doenças cardiovasculares vêm aumentando significantemente em sua incidência, passando a ser considerada um grave problema de saúde pública. Nos dias atuais, tornou-se comum casos de hipertensão arterial sistêmica, sendo considerados reflexo de um novo estilo de vida adotado pela sociedade. Dessa forma, por ter uma elevada incidência e por ser uma doença silenciosa, a maioria dos portadores não realizam ações preventivas e tem uma enorme dificuldade de adesão ao tratamento, levando, assim, a um maior número de óbitos.

De acordo com a Sociedade Brasileira de hipertensão, $30 \%$ da população brasileira pode ser considerada hipertensa, desse total, 5\% são crianças e adolescentes, e um dado preocupante é a estimativa de que cerca de 15 milhões de hipertensos desconheça sua condição e que, anualmente, quase trezentas mil pessoas morrem no Brasil por doenças cardiovasculares, onde metade dessas são decorrentes da hipertensão (SBC; SBH; SBN, 2010)

Segundo Brunner e Suddarth (2009) a hipertensão arterial sistêmica (HAS) é caracterizada como resultado de uma alteração no débito cardíaco, uma modificação na resistência periférica ou ambas. É considerado hipertenso o paciente com pressão arterial (PA) sistólica superior a $140 \mathrm{mmHg}$ e pressão diastólica superior a $90 \mathrm{mmHg}$, valores obtidos em, no mínimo, duas ocasiões distintas. O estilo de vida que as pessoas têm adotado é o principal responsável por essa situação, no qual, crianças e adolescentes comem de modo inadequado, e ainda, o uso excessivo da televisão estimula a inatividade física e eles estão expostos a sedução do tabagismo (SANTOS. et al., 2009).

Conforme Oliveira et al. (2008) a terapia antihipertensiva é extremamente eficaz em reduzir a pressão arterial, contudo, o percentual de pacientes que alcançam os alvos terapêuticos é baixo. A não adesão ao tratamento é um dos mais importantes problemas enfrentados, pois gera custos substanciais pelas baixas taxas de controle alcançados em todo mundo, o que acaba aumentando a morbimortalidade decorrente da patologia.

De acordo com Souza et al (2009), apud Santos et al (2005) a não adesão a terapêutica é complexa, pois existem diversos fatores que interferem na adesão bem como idade, sexo, etnia, escolaridade, estado civil, nível sócio econômico, crenças, hábitos culturais e de vida, autoestima, e um dos mais importantes obstáculos, a ausência de sintomas.

Segundo Figueiredo (2010), o tratamento da hipertensão pode ser com ou sem medicamento, sendo que o primeiro é realizado através do controle do peso, prática de exercício físico, melhora no padrão alimentar, dieta hipossódica, moderação no consumo de bebidas alcoólicas e abstenção do tabagismo, já o tratamento medicamentoso requer o uso de drogas prescritas pelo médico, conforme a gravidade do caso.

A falta de compromisso com o tratamento por parte dos pacientes hipertensos, gera diversas consequências na qualidade de vida do portador, pois, apesar de ser um problema de saúde pública, e por ter uma alta incidência de complicações, ainda existem aqueles que acreditam que não se faz necessário a adesão ao tratamento, deixando para intervir apenas quando a doença toma proporções mais graves levando à cardiopatias decorrentes da hipertensão. As equipes de saúde da família muitas vezes encontram entraves para que os pacientes possam aderir ao tratamento da doença. Contribuindo, assim, na identificação das principais dificuldades enfrentadas pelos portadores de hipertensão. Deste modo, objetivou-se identificar as dificuldades enfrentadas pelo portador de hipertensão arterial sistêmica a adesão do tratamento pela equipe da Estratégia Saúde da Família (ESF), avaliando a percepção dos hipertensos quanto ao seu tratamento; identificando os índices de não adesão ao tratamento medicamentoso e não medicamentoso e averiguando se o portador de HAS possui alguma complicação decorrente da doença.

\section{MATERIAL E MÉTODOS}

Trata-se de um estudo de campo, do tipo exploratório, descritivo, com abordagem quantitativa dos dados.

O estudo foi realizado no município de Cajazeiras, Paraíba, que encontra-se situado no oeste paraibano, distante $477 \mathrm{~km}$ da capital João Pessoa. Ocupa uma área de $586,275 \mathrm{~km}^{2}$, dos quais $28,193 \mathrm{~km}^{2}$ estão em perímetro urbano. Sua população recenseada pelo Instituto Brasileiro de Geografia e Estatística (2010) é de 58.437 habitantes, sendo o oitavo mais populoso do estado e o primeiro de sua microrregião. O Campo do estudo foi constituído das 12 Unidades de Saúde da Família (USF) da zona urbana do município de Cajazeiras (PB), onde são ofertados serviços como atenção a saúde da mulher, saúde da criança, vacinação, consulta de HiperDia, e atendimento ambulatorial.

A população foi composta por 4.772 hipertensos cadastrados no programa HiperDia das referidas unidades, e a amostra foi constituída de 100 hipertensos, através de uma amostra não-probabilística. Os sujeitos para a amostra da pesquisa foram selecionados obedecendo aos seguintes critérios de inclusão: ser hipertenso, não aderir ao tratamento, aceitar espontaneamente participar da pesquisa, assinar o Termo de Consentimento Livre e Esclarecido (TCLE). E como critérios de exclusão: não ser hipertenso, aderir ao tratamento, se recusar a participar do estudo e não assinar o TCLE.

Foi utilizado como instrumento de coleta de dados um questionário estruturado, composto por duas partes, a primeira contemplando dados sociodemográficos da pesquisa e a segunda atendendo aos objetivos da pesquisa.

Os dados foram coletados conforme $\mathrm{o}$ cronograma e após aprovação do Comitê de Ética em Pesquisa (CEP) da Faculdade Santa Maria. Para efetivação da coleta dos dados aguardou-se o parecer do CEP e ainda procedeu-se solicitação da coordenação do Curso Bacharelado de Enfermagem, através de ofício. Os dados foram coletados diretamente com os participantes da pesquisa, na residência dos mesmos, ou na USF nos turnos manhã e tarde, de acordo com a disponibilidade de cada participante.

Para a realização da pesquisa foram respeitados os princípios éticos e legais vinculados à pesquisa envolvendo seres humanos, contidos na Resolução $n^{\circ} 466$, 
de 12 de dezembro de 2012, do Conselho Nacional de Saúde/ CNS, mediante deferimento do Protocolo Ético, através do registro número 530.639. Para tanto, foi elaborado e incluído neste estudo o TCLE que resguarda a autonomia dos sujeitos da pesquisa, com preservação do anonimato e sigilo com relação às informações concedidas (BRASIL, 2012).

\section{RESULTADOS E DISCUSSÃO}

Para os dados sóciodemograficos houve uma pequena prevalência do gênero feminino $(65 \%)$ (Tabela 01), o que contradiz os dados da VI Diretriz Brasileira de Hipertensão ao afirmar que a prevalência global da hipertensão é semelhante entre mulheres e homens (SBC, SBH, SBN, 2010).

Corroborando com este pensamento Zaitune (2006) afirma que as mulheres geralmente têm maior percepção das doenças, e apresentam maior tendência para o autocuidado. buscando mais assistência médica do que homens, o que aumenta a probabilidade de ter a hipertensão arterial diagnosticada diferentemente dos homens.

Quanto ao estado civil, 55\% se declararam ser casados, $10 \%$ solteiro, $11 \%$ divorciado/separado, $22 \%$ viúvo e $2 \%$ união estável (Tabela 1). De acordo com a VI Diretrizes Brasileira de Hipertensão (2010) a presença de laços familiares estáveis está diretamente relacionado com o acesso aos serviços de saúde tendo em vista que existe uma maior possibilidade de diagnóstico, o que justificaria o achado de maior prevalência de hipertensos nessa classe civil.

Outra variável estudada foi a etnia, a maioria é constituída por pessoas que se consideram pardas 53\%, seguido por negros $31 \%$, e $16 \%$ consideram-se brancos (Tabela 1). A etnia negra constitui-se um fator predisponente a HAS, pode-se dizer que esse fato dar-se porque a população da cidade de Cajazeiras, tem maior prevalência de pardos e uma pouca incidência de pessoas negras. No tocante do ponto de vista racial, evidências apontam que o individuo negro tem uma maior sensibilidade ao sódio e consequentemente poderão desenvolver quadro de hipertensão em maiores proporções que indivíduos brancos (PEREIRA; SILVA, 2011).

Em relação ao grau de escolaridade a prevalência foi do fundamental incompleto (42\%), seguido de analfabeto $24 \%$, médio completo $17 \%$, médio incompleto $8 \%$, superior completo $5 \%$, superior incompleto $3 \%$, fundamental completo $1 \%$ (Tabela 1 ).

Tabela 1. Descrição demográfica de hipertensos atendidos pela equipe de saúde da família no município de Cajazeiras, Paraíba

\begin{tabular}{|c|c|c|c|c|}
\hline & & f & $\%$ & $\mathrm{p}$ \\
\hline \multirow[t]{2}{*}{ Gênero } & Masculino & 35 & 35,0 & \multirow[t]{2}{*}{$<0,05$} \\
\hline & Feminino & 65 & 65,0 & \\
\hline \multirow{5}{*}{ Estado Civil } & Solteiro & 10 & 10,0 & \multirow{5}{*}{$<0,05$} \\
\hline & Casado & 55 & 55,0 & \\
\hline & Divorciado/separado & 11 & 11,0 & \\
\hline & Viúvo & 22 & 22,0 & \\
\hline & União estável & 2 & 2,0 & \\
\hline \multirow{3}{*}{ Etnia } & Branco & 16 & 16,0 & \multirow{3}{*}{0,08} \\
\hline & Pardo & 53 & 53,0 & \\
\hline & Negro & 31 & 31,0 & \\
\hline \multirow{7}{*}{ Grau de Escolaridade } & Fundamental Completo & 1 & 1,0 & \multirow{7}{*}{$<0,01$} \\
\hline & Fundamental Incompleto & 42 & 42,0 & \\
\hline & Médio Completo & 17 & 17,0 & \\
\hline & Médio Incompleto & 8 & 8,0 & \\
\hline & Superior Completo & 5 & 5,0 & \\
\hline & Superior Incompleto & 3 & 3,0 & \\
\hline & Analfabeto & 24 & 24,0 & \\
\hline \multirow{10}{*}{ Profissão } & Do lar & 8 & 8,0 & \multirow{10}{*}{$<0,01$} \\
\hline & Aposentado & 45 & 45,0 & \\
\hline & Pensionista & 2 & 2,0 & \\
\hline & Policial & 5 & 5,0 & \\
\hline & Agricultor & 19 & 19,0 & \\
\hline & Doméstica & 13 & 13,0 & \\
\hline & Vendedora & 2 & 2,0 & \\
\hline & Estudante & 2 & 2,0 & \\
\hline & Funcionário público & 2 & 2,0 & \\
\hline & Padagoga & 2 & 2,0 & \\
\hline Idade & Média (DP) & & $59,35(14,72)$ & \\
\hline
\end{tabular}


Oliveira (2007) afirma que o baixo nível de escolaridade se torna mais difícil no processo de adesão, principalmente em pacientes idosos, que sentem mais dificuldade para diferenciar as medicações, bem como conhecer o tratamento e se dedicarem, mudando assim os hábitos adquiridos em toda uma vida.

Segundo Pires e Silva (2011) a deficiência na formação escolar pode acarretar em dificuldades relativas a assimilação de orientações dispensadas pela equipe multiprofissional o que leva a uma maior percepção dos possíveis agravos a saúde.

Em relação à profissão, a maior incidência foi de aposentados, $45 \%$, o que se justifica pela maior incidência de hipertensos considerados idosos, dessa forma, não exercem mais nenhum tipo de profissão, tendo como renda a aposentadoria.

A média de idade da amostra ficou em 59,35 anos $(\mathrm{DP} \%=14,72)$, comprovando, assim, que quanto maior a idade do indivíduo, maior é a probabilidade do mesmo adquirir a HAS (Tabela 1 ).

A hipertensão arterial aumenta linearmente com o aumento da idade, a partir da sexta década de vida o principal componente é o aumento da pressão diastólica, o risco relativo de desenvolver doença cardiovascular associado ao aumento da PA não diminui com o avanço da idade e o risco absoluto aumenta marcadamente (DBHV, 2006). Para o tempo de HAS foi observado média de 8,63 anos $(\mathrm{DP}=6,80)$. Das principais queixas das pessoas com HAS a que mais se destacou foi a cefaleia com $68 \%$ das pessoas relatando este problema, e a menos frequente foi a tosse com $19 \%$ das pessoas, além dessas outras queixas também apresentaram significância estatística: náuseas, com 38\% e dispneia com 35\% (tabela 2).

A HAS raramente manifesta sintomas ou desconforto físico, e isso constitui uma das razões do portador não se comprometer com as condutas necessárias para seu controle, pois as pessoas não se consideram doentes até o momento em que haja alterações na qualidade de vida que lhes impeçam de exercer suas funções diárias ou atividades rotineiras (SANTOS et al, 2008).

Apesar da hipertensão ser considerada uma doença silenciosa, alguns sintomas são bem característicos quando ocorre o aumento da PA, dentre eles destacam-se a cefaleia, tosse seca, náuseas e dispneia. Dessa forma, é a partir dessa sintomatologia que é possível identificar um possível quadro de aumento da pressão arterial, e para confirmação faz-se necessário a avaliação dos níveis pressóricos.

Quando os sinais e sintomas específicos aparecem, eles geralmente indicam lesão vascular, com manifestações especificas relacionadas com os órgãos servidos pelos vasos afetados (SMELTZER; BARE, 2009).

Tabela 2. Principais queixas de hipertensos atendidos pela equipe de saúde da família no município de Cajazeiras, Paraíba

\begin{tabular}{|c|c|c|c|c|}
\hline & & $\mathbf{f}$ & $\%$ & $\mathbf{p}$ \\
\hline \multirow[t]{2}{*}{ Cefaleia } & Sim & 68 & 68,0 & \multirow{2}{*}{$<0,05$} \\
\hline & Não & 32 & 32,0 & \\
\hline \multirow[t]{2}{*}{ Insônia } & Sim & 51 & 51,0 & \multirow{2}{*}{0,89} \\
\hline & Não & 49 & 49,0 & \\
\hline \multirow[t]{2}{*}{ Ansiedade } & Sim & 53 & 53,0 & \multirow{2}{*}{0,57} \\
\hline & Não & 47 & 47,0 & \\
\hline \multirow[t]{2}{*}{ Náuseas } & Sim & 38 & 38,0 & \multirow{2}{*}{$<0,05$} \\
\hline & Não & 62 & 62,0 & \\
\hline \multirow[t]{2}{*}{ Tosse } & Sim & 19 & 19,0 & \multirow{2}{*}{$<0,01$} \\
\hline & Não & 81 & 81,0 & \\
\hline \multirow[t]{2}{*}{ Tontura } & Sim & 49 & 49,0 & \multirow{2}{*}{0,89} \\
\hline & Não & 51 & 51,0 & \\
\hline \multirow[t]{2}{*}{ Dispneia } & Sim & 35 & 35,0 & \multirow{2}{*}{$<0,05$} \\
\hline & Não & 65 & 65,0 & \\
\hline \multirow[t]{2}{*}{ Visão turva } & Sim & 41 & 41,0 & \multirow{2}{*}{0,89} \\
\hline & Não & 59 & 59,0 & \\
\hline
\end{tabular}

A tabela 3 expõe os principais motivos de não aderirem ao tratamento. $\mathrm{O}$ motivo mais relatado foi a falta de conhecimento com $89 \%$ ( $p<0,01)$ e os dois menos frequentes foram, respectivamente as dificuldades financeiras $(4 \%)$ e medo do efeito do medicamento $(9 \%)$.

Em relação a esses motivos, a grande maioria dos hipertensos desconhecem o que é a HAS, quais suas possíveis complicações como é o exemplo da insuficiência renal dislipidemia, associação com o DM, infarto agudo do miocárdio (IAM), angina, dentre outras patologias, fazendo com que o mesmo não realize medidas de prevenção e tratamento, levando assim ao agravamento da doença.

Segundo Santos et al (2008) quanto maior o grau de conhecimento do indivíduo sobre seu problema, maior é a possibilidade de seu comprometimento efetivo no autocuidado e, portanto, maior chance de sucesso.

Outro dado significante é a falta de orientação $52 \%$ esse dado esta diretamente associado à falta de ações educativas por parte da equipe multidisciplinar, que tem como finalidade orientar o paciente quanto a importância da realização de um tratamento adequado. 
As sessões educativas devem ser permeadas pela educação em saúde, possibilitando assim mudanças de comportamento para o exercício da cidadania, esta mudança é viabilizada quando o profissional de saúde reconhece e valoriza a importância do saber construído pelo paciente, dessa forma a partir desse reconhecimento, ocorrerá a constituição efetiva de novos conhecimentos, modificando o comportamento da saúde da clientela, objetivando atingir o melhor nível de bem estar (SANTOS, et al. 2008)

Tabela 3. Motivos que impedem de aderir ao tratamento de hipertensos atendidos pela equipe de saúde da família no município de Cajazeiras, Paraíba

\begin{tabular}{|c|c|c|c|c|}
\hline & & $\mathbf{F}$ & $\%$ & $\bar{p}$ \\
\hline \multirow{2}{*}{ Falta de Conhecimento } & Sim & 89 & 89,0 & \multirow{2}{*}{$<0,01$} \\
\hline & Não & 11 & 11,0 & \\
\hline \multirow{2}{*}{ Falta de Orientação } & Sim & 52 & 52,0 & \multirow{2}{*}{0,81} \\
\hline & Não & 48 & 48,0 & \\
\hline \multirow{2}{*}{ Medo do Efeito medicamentoso } & Sim & 9 & 9,0 & \multirow{2}{*}{$<0,01$} \\
\hline & Não & 91 & 91,0 & \\
\hline \multirow{2}{*}{ Dificuldades Financeiras } & Sim & 4 & 4,0 & \multirow{2}{*}{$<0,01$} \\
\hline & Não & 96 & 96,0 & \\
\hline \multirow{2}{*}{ Dificuldade de acesso ao serviço } & Sim & 17 & 17,0 & \multirow{2}{*}{$<0,05$} \\
\hline & Não & 83 & 83,0 & \\
\hline
\end{tabular}

$\mathrm{Na}$ tabela 4, observa-se os resultados relacionados a casos de antecedentes familiares, sendo que, $84 \%$ relataram que sim. Os mais frequentes foram os irmãos, com $66 \%$ dos casos, e o que menos se destacou foi avó com $10 \%$ dos casos. Sabe-se que os antecedentes familiares correspondem a um fator impossível de ser modificado, dessa forma, faz-se necessário a monitorização do individuo, uma vez que o mesmo possua histórico familiar de HAS, pois esses indivíduos tem um grande risco de desenvolve-la no futuro.

Uma pesquisa do Ministério da Saúde revelou que no período de 2006-2009, a doença aumentou em todas as faixas etárias, porém a prevalência continua sendo de idosos. As estimativas de hereditariedade, mostram que a hipertensão em indivíduos com um dos genitores afetados é de 20 a $40 \%$.

De acordo com a VI Diretrizes Brasileira de Hipertensão (2010) a contribuição dos fatores genéticos para a gênese da hipertensão, esta bem estabelecida na população, porém, não existe até o momento, variantes genéticas que possam ser utilizadas para predizer o risco individual de se desenvolver a HAS (SBC; SBH; SBN, 2010).

Tabela 4. Casos de antecedentes familiares de hipertensos atendidos pela equipe de saúde da família no município de Cajazeiras, Paraíba

\begin{tabular}{|c|c|c|c|c|}
\hline & & $\bar{F}$ & $\%$ & $\mathbf{p}$ \\
\hline \multirow{2}{*}{$\begin{array}{l}\text { Possui antecedentes Familiares de } \\
\text { HAS }\end{array}$} & Sim & 84 & 84,0 & \multirow{2}{*}{$<0,05$} \\
\hline & Não & 16 & 16,0 & \\
\hline \multirow{2}{*}{ Pai } & Sim & 39 & 39,0 & \multirow{2}{*}{$<0,05$} \\
\hline & Não & 61 & 61,0 & \\
\hline \multirow{2}{*}{ Mãe } & Sim & 51 & 51,0 & \multirow{2}{*}{0,89} \\
\hline & Não & 49 & 49,0 & \\
\hline \multirow{2}{*}{ Avó } & Sim & 10 & 10,0 & \multirow{2}{*}{$<0,01$} \\
\hline & Não & 90 & 90,0 & \\
\hline \multirow{2}{*}{ Avô } & Sim & 8 & 8,0 & \multirow{2}{*}{$<0,01$} \\
\hline & Não & 92 & 92,0 & \\
\hline \multirow{2}{*}{ Tios } & Sim & 27 & 27,0 & \multirow{2}{*}{$<0,05$} \\
\hline & Não & 73 & 73,0 & \\
\hline \multirow{2}{*}{ Irmãos } & Sim & 66 & 66,0 & \multirow{2}{*}{$<0,05$} \\
\hline & Não & 34 & 34,0 & \\
\hline
\end{tabular}

Na tabela 5 verifica-se que $32 \%$ dos hipertensos relataram ter histórico de outra doença, o diabetes mellitus (DM) obteve uma maior prevalência 32\%, seguido por dislipidemia $13 \%$ e doenças cardiovasculares 5\%. Essa associação dar-se por existir uma correlação da hipertensão com outras patologias, esse fato ocorre principalmente quando o usuário não adota medidas de controle e tratamento adequado da HA, levando assim a complicações que são decorrentes da gravidade e cronificação da HAS.

A hipertensão também pode ser responsável pelo surgimento de comorbidades a exemplo de doenças coronarianas, Acidentes Vasculares Encefálicos (AVE), insuficiência renal, doenças vasculares periféricas dentre outras. Essas comordidades são decorrentes das complicações da HAS e se diferem dos fatores de risco que são condições comportamentais, as quais contribuem 
para o desenvolvimento da doença. Devido a alta prevalência, associada ao alto índice de mortalidade, fazse necessário uma atenção a prevenção afim de evitar o desenvolvimento de novos casos ou que os existentes evoluam para quadros mais graves (MACHADO et al., 2012)
Quanto a frequência de idas a USF, $46 \%$ relataram ir uma vez ao mês e $24 \%$ relataram ir uma vez a cada seis meses (Tabela 5). Dessa forma, o presente estudo mostra uma considerável taxa de regularidade ao serviço de saúde, destacando os pacientes, mesmo não seguindo corretamente todas as recomendações propostas, ainda assim se preocupam com a doença.

Tabela 5. Relacionados da Unidade Básica de Saúde e os pacientes de hipertensos atendidos pela equipe de saúde da família no município de Cajazeiras, Paraíba

\begin{tabular}{|c|c|c|c|c|}
\hline & & $\overline{\mathbf{f}}$ & $\%$ & $\overline{\mathbf{p}}$ \\
\hline \multirow{4}{*}{$\begin{array}{l}\text { Histórico de Outra } \\
\text { doença }\end{array}$} & Não & 50 & 50,0 & \multirow{4}{*}{$<0,05$} \\
\hline & Dislipidemia & 13 & 13,0 & \\
\hline & Diabetes melitus & 32 & 32,0 & \\
\hline & Doenças cardiovasculares & 5 & 5,0 & \\
\hline \multirow{5}{*}{$\begin{array}{l}\text { Frequência que vai a } \\
\text { UBSF }\end{array}$} & Uma vez no mês & 46 & 46,0 & \multirow{5}{*}{$<0,05$} \\
\hline & Mais de uma vez no mês & 4 & 4,0 & \\
\hline & Passa mais de dois meses sem ir a unidade & 16 & 16,0 & \\
\hline & Vai de seis em seis meses & 24 & 24,0 & \\
\hline & Nenhuma vez & 10 & 10,0 & \\
\hline \multirow{4}{*}{$\begin{array}{l}\text { Relação com os } \\
\text { profissionais }\end{array}$} & Boa & 58 & 58,0 & \multirow{4}{*}{$<0,01$} \\
\hline & Razoável & 31 & 31,0 & \\
\hline & Ruim & 9 & 9,0 & \\
\hline & Péssima & 2 & 2,0 & \\
\hline \multirow{3}{*}{$\begin{array}{l}\text { Dificuldade em receber a } \\
\text { medicação }\end{array}$} & Sim & 34 & 34,0 & \multirow{3}{*}{0,18} \\
\hline & As vezes & 28 & 28,0 & \\
\hline & Nunca & 38 & 38,0 & \\
\hline \multirow{2}{*}{$\begin{array}{l}\text { Possui Complicações } \\
\text { decorrentes da doença }\end{array}$} & Sim & 27 & 27,0 & \multirow{2}{*}{$<0,05$} \\
\hline & Não & 73 & 73,0 & \\
\hline \multirow{2}{*}{ IAM } & Sim & 4 & 4,0 & \multirow{2}{*}{$<0,01$} \\
\hline & Não & 96 & 96,0 & \\
\hline \multirow{2}{*}{ AVE } & Sim & 17 & 17,0 & \multirow{2}{*}{$<0,01$} \\
\hline & Não & 83 & 83,0 & \\
\hline \multirow{2}{*}{ Distúrbios Circulatórios } & Sim & 8 & 8,0 & \multirow{2}{*}{$<0,01$} \\
\hline & Não & 92 & 92,0 & \\
\hline \multirow{2}{*}{ Prática Atividade Física } & Sim & 11 & 11,0 & \multirow{2}{*}{$<0,01$} \\
\hline & Não & 89 & 89,0 & \\
\hline \multirow{2}{*}{ Segue Dieta } & Sim & 8 & 8,0 & \multirow{2}{*}{$<0,01$} \\
\hline & Não & 92 & 92,0 & \\
\hline \multirow{2}{*}{ Faz uso da medicação } & Sim & 56 & 56,0 & \multirow{2}{*}{0,56} \\
\hline & Não & 44 & 44,0 & \\
\hline
\end{tabular}

Segundo Faquinello et al. (2010) o acompanhamento das pessoas com diagnóstico de HAS é realizado pela ESF atuante na USF, que deve ser localizada na região mais próxima da residência do paciente, pois se entende que para otimização do atendimento, o primordial é que essas unidades de assistência sejam de fácil acesso e próximo a residência do usuário, a USF deve ser a primeira referência de apoio formal procurada pela população para o acompanhamento e prevenção de saúde, bem como, para retirar dúvidas no que se diz respeito a patologia.

Sobre a relação com o atendimento à saúde, $58 \%$ relataram um bom relacionamento com os profissionais e apenas $2 \%$ relataram como péssima essa relação. Esse resultado pode estar associado, também, a falta de conhecimento do paciente, pois o mesmo acredita que o papel do profissional se restringe apenas a entrega de medicação e consulta médica quando, na verdade, é importante também para os usuários, além das ações supracitadas, que o profissional adote medidas de acolhimento, desenvolva ações que envolvam identificação dos problemas e principalmente medidas de resolutividade para os mesmos.

Em relação às dificuldades em receber a medicação, 38\% afirmam nunca ter tido essa dificuldade, seguida por $34 \%$ que relataram ter dificuldades em receber os medicamentos, e as vezes surge alguma dificuldade $28 \%$.

Segundo Paula et al. (2011), na sociedade brasileira, os problemas assinalados com respeito ao acesso são significativamente importantes, em especial quando se leva em consideração a HAS. De acordo com a estimativa do Ministério da Saúde, dos 16,8 milhões de brasileiros que sofrem de hipertensão arterial, somente 7,7 milhões estão cadastrados no Sistema Único de Saúde (SUS). Logo, o acesso aos medicamentos usados para 
tratar tal enfermidade fica comprometido, uma vez que, apesar de estar garantido constitucionalmente, nem todos os pacientes têm esse acesso viabilizado.

Para as possíveis complicações da decorrente doença, observa-se na tabela 5 que $27 \%$ possuem essas complicações, sendo 4\% com IAM, 17\% com AVE, $8 \%$ distúrbios circulatórios. Essas complicações decorrem da falta de tratamento adequado, bem como de medidas preventivas para se evitar complicações, um dos fatores que levam a esse quadro é a ausência de sintomas da doença, o que pode levar a uma falsa ilusão de que a doença está controlada, levando assim a complicações graves como AVE, IAM, distúrbios circulatórios dentre outros.

A HAS é apontada como fator de risco para complicações e doenças cardiovasculares na sociedade atual, tais como morte súbita, edema agudo de pulmão, insuficiência renal, IAM e AVE, explicando 54\% das mortes por acidente vascular encefálico e $47 \%$ daquelas por doença isquêmica do coração (SANTOS; MOREIRA, 2012).

Em relação a prática de atividades física, apenas $11 \%$ declararam praticar, enquanto a maioria, $89 \%$ não realizam nenhum tipo de atividade. Em relação à dieta, apenas $8 \%$ seguem adequadamente. Essas afirmativas demonstram que o paciente hipertenso não faz uso da terapia não medicamentosa que envolve mudanças no estilo de vida, que também é considerado um fator muito importante para a regularização da PA, bem como através dessa mudança de estilo de vida os fatores de risco modificáveis podem ser alterados levando assim a uma redução dos níveis pressóricos.

As mudanças de comportamentos necessárias para o controle da PA são desafiadoras para hipertensos e serviços de saúde. Estudos que identifiquem as prevalências de adesão às diferentes modalidades de tratamento e os grupos populacionais mais vulneráveis à não adesão são importantes para direcionar ações individuais e coletivas de atenção à saúde (GIROTTO, et al. 2013).

No que se diz respeito ao uso adequado da medicação, a maioria (56\%) faz uso de medicação, esse maior percentual pode ser explicado pelo fato de que os pacientes vêm na terapia medicamentosa uma maior chance de redução dos níveis pressóricos. Enquanto 44\% não utilizam a medicação de forma correta, o que pode estar associado a ausência de sintomas, esquecimento, ou até mesmo por efeitos colaterais da medicação.

Segundo Vito et al. (2011), o tratamento da hipertensão envolve medicação adequada associada a mudanças no estilo de vida, esta combinação contribui para uma diminuição de eventos cardiovasculares fatais e não fatais, além de evitar o elevado custo social de seu tratamento e de suas complicações, favorecendo assim uma melhor qualidade de vida aos pacientes portadores de HAS.

\section{CONCLUSÕES}

Existe uma predominância do gênero feminino de usuários que não aderem à terapia para HAS, com baixo grau de escolaridade, etnia parda, em sua maioria, casados, aposentados.
As principais queixas apresentadas pelos pacientes tratados são a cefaleia, seguida de insônia e ansiedade. E os motivos que os levam a não aderir a terapêutica instituída são a falta de conhecimento, a prática de atividades físicas e adesão a uma dieta adequada.

Há necessidade de investimentos maciços nos processos de trabalho que envolvam a educação em saúde como estratégia que garantes resultados satisfatórios na promoção e proteção à saúde dos indivíduos.

\section{REFERÊNCIAS}

BRASIL. Ministério da Saúde. Conselho Nacional de saúde. Comissão Nacional em Pesquisa. Normas para Pesquisa Envolvendo Seres Humanos (Res. CNS. 466/2012). Brasília; 2012.

BRASIL. Ministério da Saúde. Manual de condutas Médicas: Hipertensão arterial e Diabetes Mellitus. Brasília: Ministério da saúde, 2002.

BRASIL. Ministério da Saúde. Cadernos de atenção básica: Hipertensão arterial sistêmica. Brasília: Ministério da saúde, 2005.

BRASIL. Ministério da Saúde. Cadernos de atenção básica: Hipertensão arterial sistêmica. Brasília: Ministério da saúde, 2006.

BRASIL. Organização Pan-Americana da saúde. Avaliação do plano de reorganização da atenção à hipertensão arterial e ao diabetes mellitus no Brasil. Brasília, 2007.

BRASIL. Ministério da saúde. Política Nacional da Atenção Básica. v.4, 4 ed. Brasília, 2007.

BRASIL. Plano de reorganização da atenção à hipertensão e ao diabetes mellitus. Brasília, 2008.

BRASIL. Ministério da Saúde. A Experiência Brasileira em Sistemas de Informação em Saúde, fundação Oswaldo cruz. Brasília, 2009.

BRUNNER, S.; SUDDARTH, B. Tratado de enfermagem médico cirúrgica. Rio de Janeiro: Guanabara Koogan, 2009

CENTRO Hiperdia: Fundação Imepen. Fundação Instituto Mineiro de Estudos e Pesquisas em Nefrologia. Disponível em: <http://www.imepen.com/hiperdia/>. Acesso em: 16 out. 2013.

CHAVES, E. S., LÚCIO, I. M. L.; ARAÚJO, T. L.; DAMASCENO, M. M. C. Eficácia de programas de educação para adultos portadores de hipertensão arterial. Rev. Brasileira de enfermagem, v.59, n.4, p.543-547. São Paulo, 2006.

DBHA VI. VI Diretrizes Brasileira de Hipertensão. Sociedade Brasileira de Hipertensão: Revista Hipertensão. 
v. 13, n. 1, p. 8-61, 2010.

DBHA VI. VI Diretrizes Brasileira de Hipertensão. Sociedade Brasileira de Hipertensão: Revista Hipertensão. v. 5, n. 1, p. 7, 2006.

FAQUINELLO, A.; CARREIRA, L.; MARCON, S. S. A unidade básica de saúde e sua função na rede de apoio social ao hipertenso. Texto Contexto Enferm, v.19, n.4 p.736-744. Florianópolis, 2010.

FIGUEIREDO, N. N; ASAKURA, L. Adesão ao tratamento anti-hipertensivo: dificuldades relatas por indivíduos hipertensos. São Paulo: UNIFESP, 2010.

FILHA, F. S. S. C; NOGUEIRA, L. T; VIANA, L. M. M. Hiperdia: Adesão e Percepção de usuários acompanhados pela estratégia saúde da família. Rene: Fortaleza, p.930936, 2011.

GIROTTO, E.; ANDRADE, S. M.; CABRERA, M. A. S.; MATSUO, T. Adesão ao tratamento farmacológico e não farmacológico e fatores associados na atenção primaria da hipertensão arterial. Ciência \& Saúde Coletiva, v.18, n.6, p.1763-1772. São Paulo, 2013.

IBGE. Instituto Brasileiro de Geografia Estatística. IBGE Cidades. Brasília. Ministério do Planejamento, Orçamento e Gestão, 2009. Disponível em: http://www.ibge.gov.br/cidadesat/topwindow.htm?1acesso em: 23 de outubro de 2013.

INCA. INSTITUTO NACIONAL DO CANCER. Ministério da Saúde. Secretaria de Atenção Básica. Secretaria de Vigilância em Saúde. Tabagismo: Um grave problema de saúde Pública, p.26, 2007.

MANO, R. Hipertensão Arterial Sistêmica. 1.ed. Rio de Janeiro, 2009.

MACHADO, M. C; PIRES, C. G. S; LOBÃO, W. M. Concepção dos hipertensos sobre os fatores de risco para a doença. Ciência \& Saúde Coletiva, v.17, n.5, p.13651374, São Paulo, 2012

NEVES, F. A; JUNGES F. Sistema de informação em saúde como instrumento de avaliação da saúde da população. Rio Grande do Sul: UFRGS, 2010.

OLIVEIRA, T. R. T; PEDROSA, L. A. K; GONÇALVES R. M. D. A. Estudo da hipertensão arterial sistêmica: Repercussões quanto a adesão ao tratamento. Rev. Triang. Ens. Pesq. Ext, v.1, n.1, Uberaba, 2008.
PAULA, P. A. B.; STEPHAN-SOUZA, A. I.; VIEIRA, R. DE C. P. A.; ALVES, T. N. P. . O uso do medicamento na percepção do usuário do Programa Hiperdia. Ciênc. saúde coletiva, vol.16, n.5, p. 2623-2633..

PEREIRA, A. C.; KRIEGER, J. E. Hipertensão. Rev. da Sociedade Brasileira de Hipertensão, v.8, n.4. São Paulo, 2005.

PEREIRA, M. P. B.; BARCELLOS, C. O Território no programa saúde da família. Revista Brasileira de Geografia Mádica e da Saúde. Uberlândia, n1, p. 47-59, 2006.

PEREIRA, Q. T. S.; SILVA, K. S. C. G. Motivos que levarão o paciente hipertenso à abandonar o tratamento anti-hipertensivo em uma unidade de saúde. Cruz da Almas, 2011.

SANTOS, A. J. M.; ROSA, C.; OLIVEIRA, E. L.; ALMEIDA, J. R.; SCHNEIDER, R. M.; ROCHA, S. S. L.; COUTINHO, R. M. C. A não adesão de pacientes hipertensos ao tratamento em unidade básica de saúde (UBS). Rev Inst Ciênc Saúde. v.27, n.4, p.330-337. Campinas. 2009.

SANTOS, J.C; MOREIRA, T.M.M. Fatores de risco e complicações em hipertensos e diabéticos de uma regional sanitária do nordeste brasileiro. São Paulo-SP, 2012.

SMELTZER, S. C.; BARE, B. G. Tratado de enfermagem médico-cirúrgica. 9. ed. Rio de Janeiro: Guanabara Koogan, 2009.

SBC; SBH; SBN. Sociedade Brasileira de Cardiologia/Sociedade Brasileira de Hipertensão/Sociedade Brasileira de Nefrologia. VI Diretrizes Brasileiras de Hipertensão. Arq Bras Cardiol; v.95(1 supl.1): p.1-51. 2010.

SBC; SBH; SBN. Sociedade Brasileira de Cardiologia/Sociedade Brasileira de Hipertensão/Sociedade Brasileira de Nefrologia. V Diretrizes Brasileiras de Hipertensão. Arq. Bras. Cardiol. v.89 n.3 São Paulo. 2007.

SOUZA, C. B.; BATISTIN, G. G.; AMARAL, C. P.; SILVA, D. H. A.; FURBINO, S. A. R. Fatores que interferem a adesão ao tratamento da hipertensão arterial sistêmica. Governador Valadares. UNIVALE, 2009. 